MALARIA

JOURNAL

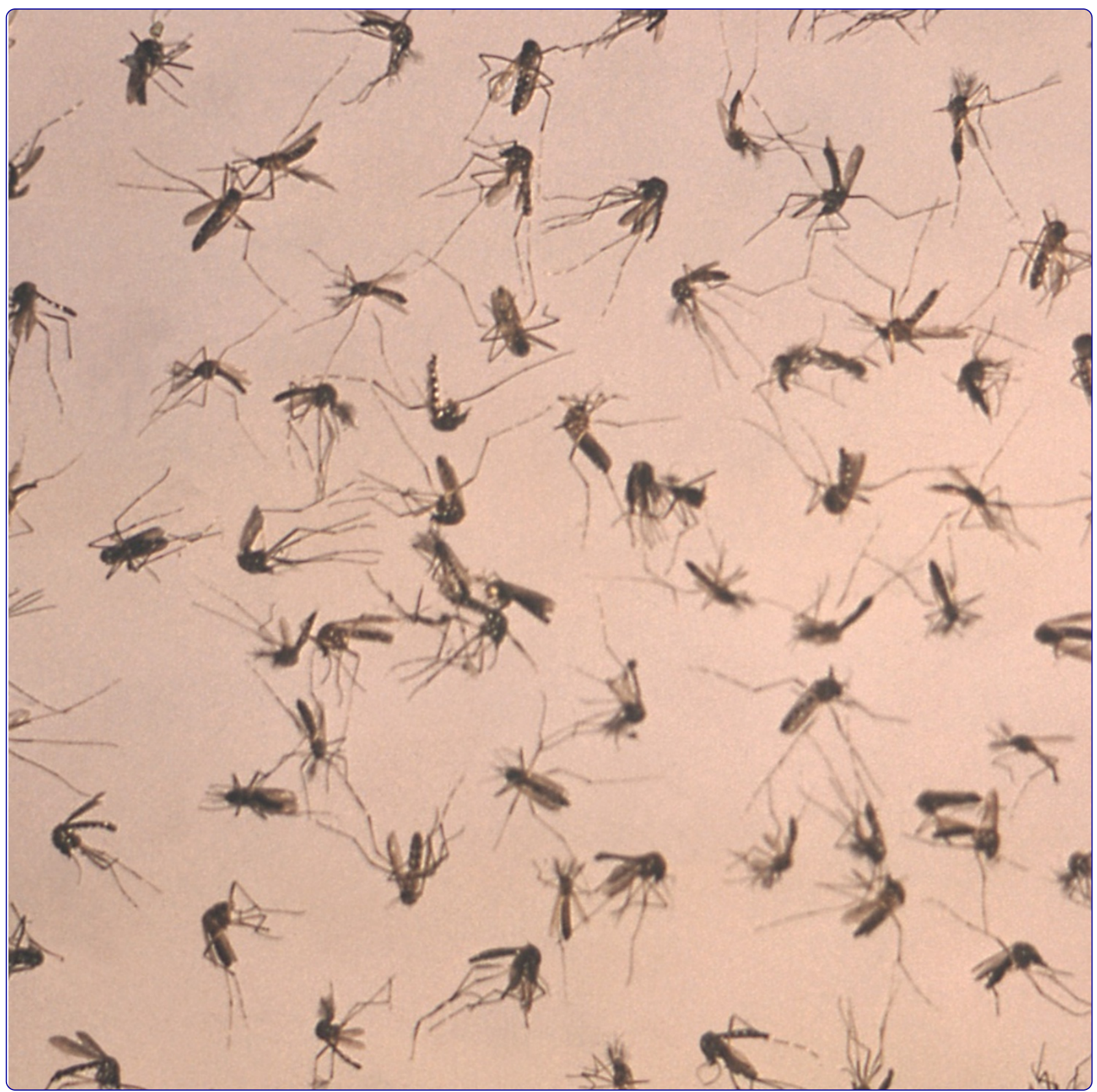

\title{
Ecological niche and potential distribution of Anopheles arabiensis in Africa in 2050
}

Drake and Beier 


\title{
Ecological niche and potential distribution of Anopheles arabiensis in Africa in $\mathbf{2 0 5 0}$
}

\author{
John M Drake ${ }^{1 *}$ and John C Beier ${ }^{2}$
}

\begin{abstract}
Background: The future distribution of malaria in Africa is likely to be much more dependent on environmental conditions than the current distribution due to the effectiveness of indoor and therapeutic anti-malarial interventions, such as insecticide-treated nets (ITNs), indoor residual spraying for mosquitoes (IRS), artemisinin-combination therapy (ACT), and intermittent presumptive treatment (IPT). Future malaria epidemiology is therefore expected to be increasingly dominated by Anopheles arabiensis, which is the most abundant exophagic mosquito competent to transmit Plasmodium falciparum and exhibits a wide geographic range.

Methods: To map the potential distribution of An. arabiensis in Africa, ecological niche models were fit to 20th century collection records. Many common species distribution modelling techniques aim to discriminate species habitat from the background distribution of environments. Since these methods arguably result in unnecessarily large Type I and Type II errors, LOBAG-OC was used to identify the niche boundary using only data on An. arabiensis occurrences. The future distribution of An. arabiensis in Africa was forecasted by projecting the fit model onto maps of simulated climate change following three climate change scenarios.
\end{abstract}

Results: Ecological niche modelling revealed An. arabiensis to be a climate generalist in the sense that it can occur in most of Africa's contemporary environmental range. Under three climate change scenarios, the future distribution of An. arabiensis is expected to be reduced by 48\%-61\%. Map differences between baseline and projected climate suggest that habitat reductions will be especially extensive in Western and Central Africa; portions of Botswana, Namibia, and Angola in Southern Africa; and portions of Sudan, South Sudan, Somalia, and Kenya in East Africa. The East African Rift Valley and Eastern Coast of Africa are expected to remain habitable. Some modest gains in habitat are predicted at the margins of the current range in South Sudan, South Africa, and Angola.

Conclusion: In summary, these results suggest that the future potential distribution of An. arabiensis in Africa is likely to be smaller than the contemporary distribution by approximately half as a result of climate change. Agreement among the three modelling scenarios suggests that this outcome is robust to a wide range of potential climate futures.

Keywords: Anopheles arabiensis, Ecological niche model, Climate change

\footnotetext{
* Correspondence: jdrake@uga.edu

'Odum School of Ecology, University of Georgia, 140 E Green Street,

30602-2202 Athens, GA, USA

Full list of author information is available at the end of the article
} 


\section{Background}

Despite substantial reductions in malaria incidence, particularly in sub-Saharan Africa [1,2], the global burden of malaria remains in the hundreds of millions of cases annually. Recent estimates range from 225 to 515 million cases per year [3-5], resulting in more than a half million deaths per year $[4,6]$. Although the decline of malaria in subSaharan Africa is generally attributed to anti-malarial interventions including the distribution of insecticidetreated nets (ITNs), artemisinin-combination therapy (ACT), intermittent presumptive treatment (IPT, [7]), and indoor residual spraying for mosquitoes (IRS), these cannot account for all observed reductions in malaria incidence [2]. For instance, malaria was observed to decline from holoendemic levels to prevalence of $30 \%$ to $50 \%$ on the island of Pemba, Tanzania prior to the onset of vector control activities [8]. An entomologic study by Meyrowitsch et al. [9] of two rural communities in the nearby Tanga region of Tanzania indicated that these declines are most likely due to declines in the abundance of Anopheles gambiae and Anopheles funestus mosquitoes, which they in turn attributed to declining precipitation and interruptions in annual (periodic) rainfall patterns [9]. These studies indicate that changes in climate and consequent weather patterns may be equally responsible for declines in African malaria, at least in some regions [9].

Despite decelerations in the emission of greenhouse gases, changes in the global climate system are now widely expected to continue to 2100 and beyond [10]. The potential consequences of these changes for human health, and the distribution of vector-borne diseases in particular, has been especially controversial [1,11-20]. The recent surge in anti-malarial activity has given rise to a new question: What will be the future distribution of malaria, given that both entomologic and epidemiologic circumstances are changing? Among other anticipated effects of these changes, it seems probable that future human cases of malaria in Africa will be disproportionately due to the vector Anopheles arabiensis compared with historical patterns because the historically dominant vectors, An. gambiae and An. funestus, are selectively targeted by indoor interventions [21] and in many cases are declining in relative abundance $[9,22,23]$. Anopheles arabiensis, by contrast, exhibits greater behavioral plasticity, is more associated with outdoor habitats, and is more likely to bite susceptible persons out of doors (exophagy) where protections are less likely to be in place [24]. Particularly, An. arabiensis is well known to favour dry (savannah) disturbed habitats [24] while larval habitats are primarily small, temporary, freshwater pools and other built features of the landscape, such as rice fields and fish ponds [24]. Additionally, An. arabiensis is more commonly found in urban environments, where an increasing proportion of sub-Saharan Africans reside, than An. gambiae [25]. Thus, interventions with ITNs are less effective against $A n$. arabiensis than An. gambiae and $A n$. funestus [26]. Further, because An. arabiensis executes its life cycle outside the built environment, it serves as a transmission route more likely to be subject to climate fluctuations. Taken together, these observations suggest that even as the transmission of malaria may be expected to continue to decline (because of aggressive interventions and continuing urbanization of the human population) that portion of transmission that remains will be disproportionately due to An. arabiensis and disproportionately subject to environmental conditions, a pattern that has been reported in lowland areas of Nyanza Province, Kenya [27].

Mapping the future potential distribution of An. arabiensis is therefore an important step to determining the future geography of malaria. Prior work has focused on modelling the microhabitat conditions conducive to mosquitoes, including land cover and human population and development over smaller areas [28]. In contrast, the expression potential distribution is used to refer to the large-scale geographic regions permissive to the persistence of a vector species in the absence of vector control, as set by conditions of the regional climate. That is, the potential distribution concerns not only the most conducive environmental conditions, but also the conditions at its environmental margins. Mapping the probable effects of climate change on the distribution of An. arabiensis therefore requires first estimating its ecological niche, the range of environmental conditions in which An. arabiensis is found. Because of its ecology, the space of these conditions is determined primarily by temperature and precipitation [29].

Many studies of species distributions seek to fit a model that discriminates the environments in which a species is found from the distribution of environments in a representative geographic region (the "background") [30-32]. Such models, commonly called "presence-background models" may be proportional to the probability of species presence [32-34], but should not be considered models of the ecological niche because niche environments are a subset of the environmental background, not a complementary set, and because not all niche environments are in fact occupied.

A hypothetical scenario illustrates the problem (Figure 1). In each of three panels are two hypothetical environmental variables. In the left most panel are points which represent sampled environmental conditions at which the species is found. These points are sampled from a bivariate Gaussian density. The bold line corresponds to probability density $p=0.002$, and represents the "true" niche boundary. The true population mean of this density is indicated by the black cross. The convex hull of these points is also drawn 


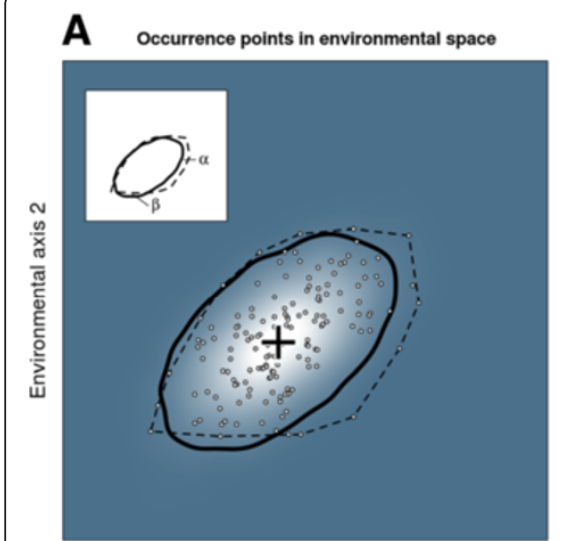

Environmental axis 1

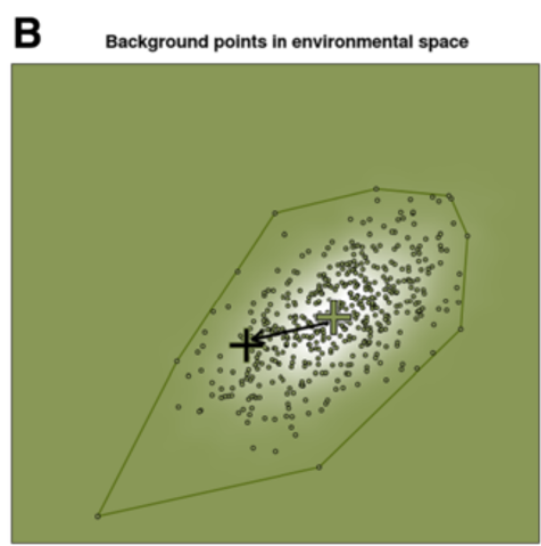

Environmental axis 1

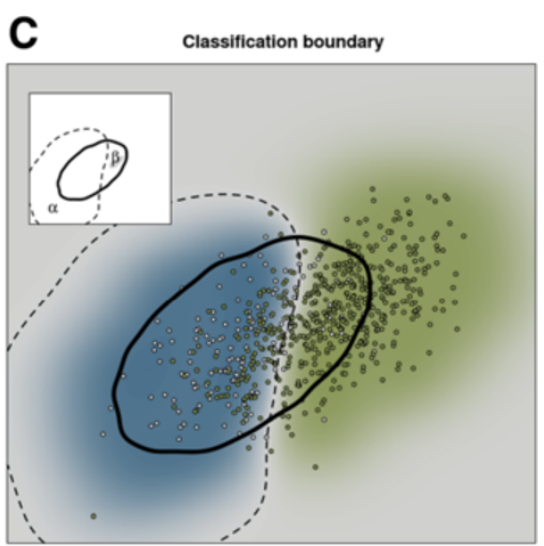

Environmental axis 1

Figure 1 Boundary estimation versus discrimative methods for ecological niche modelling. Simulated data illustrates why modelling a species'potential distribution is a problem for boundary estimation not classification. A. The left most panel represents the habitat in two environmental dimensions (e.g., precipitation and temperature) in locations at which a species is known to occur. The heavy curve depicts the true niche of the species. The dashed line is the convex hull of the sample, a naive estimate of the species niche. The black cross represents the center of the species niche, which is the most probable set of environmental conditions at which the species occurs. $\mathbf{B}$. The center panel represents samples of environmental conditions at locations taken at random from the background distribution of environments. The green cross indicates the mean environment. The arrow is a vector of "niche displacement". C. The right most panel depicts both occurrence and background data. The dashed line is the estimated optimal classification boundary between occurrence and background points. The blue-green color gradient depicts the conditional probability that a given instance is an occurrence points (blue: $P$ (occurrence) $=1$; gray: $P$ (occurrence) $=0.5$; green: $P$ (occurrence) $=0$ ). Inset plots illustrate the region of environmental space in which each fit model makes Type I $(a)$ or Type II $(\beta)$ errors.

as a "naive" model of the niche. Insofar as this naive model does not coincide with the true niche boundary it makes Type I $(\alpha)$ and Type II ( $\beta$ ) errors (inset plot). In the centre panel, plotted on the same axes, are samples of the bivariate Gaussian distribution of environmental conditions from the "background" distribution. For comparison with the first panel, a convex hull is again plotted around the set of observed background points. The true mean of this density is indicated by a green cross. The displacement between the black cross and the green cross shows that this hypothetical species is in fact selective for particular environments - it is not simply found in environments in proportion to their realization in nature. Finally, both sets of points are plotted together in the third panel. Additionally, the conditional probability that an observation sampled from any point in the space would be an occurrence or background instance is plotted with a bluegreen gradient. The classification boundary optimal for separating occurrence and background points (i.e., $P$ (occurrence $\left.\left(x_{1}, x_{2}\right)=0.5\right)$ ) is shown with a dashed line. This classification boundary is representative of the kind of model fit by presence-background estimators. The inset plot depicts the Type I and Type II errors associated with this model, clearly much larger than for the naive convex hull model.

Inspection of the figure shows why this is so: since the occurrence data are a subset of the background, the classification boundary is biased in the direction of the displacement between the niche and the background.
Intuitively, one can see there are two reasons why this boundary must be biased and why the errors must be distributed in the $\left(x_{1}, x_{2}\right)$ coordinate space in the way that they are: (1) Extreme environments in the direction of niche displacement will be assigned to the niche even when there is no evidence that these environments belong to the niche; (2) Intermediate environments in the opposite direction will be incorrectly classified as unsuitable, despite being nearly central within the niche, because the relatively frequency of these points is small compared with the vast number of background points presented to the model. What this illustration shows is that to get a good model (dashed lines) of the true niche (heavy curve) requires drawing a boundary that is, in some sense, "around" the observed occurrence points, not one that discriminates occurrence points from the background.

In conclusion, this example illustrates why ecological niche modelling should be construed as a problem for boundary identification, not classification. Indeed, regardless of how effective discriminative methods are at predicting contemporary species collections (i.e., the conditional probability of occurrence), such results should be viewed with caution when it comes to estimating potential distributions and for extrapolating to future climate scenarios, which may contain so-called no analog environments, combinations of environmental conditions not presently in existence on earth and therefore not available to learn from. 
Low bias bootstrap aggregation for one class data (LOBAG-OC) is a recently developed boundary identification method for ecological niche modelling [35]. LOBAG-OC is conceptually superior for this task, compared with habitat suitability models that return a continuous measure such as Ecological Niche Factor Analysis (ENFA) [36] or MAXENT [33,37], because LOBAG-OC estimates the niche boundary directly. This is important for two reasons. First, because ENFA, MAXENT, and related methods draw on the higher moments (mean and variance) of the observed distribution of occurrence points, they are least accurate at the range boundaries and vulnerable to biases in sampling. Second, the translation of a measure of habitat suitability into a potential range requires the determination of a "cut-off" that is rarely amenable to empirical analysis and so therefore must be determined more or less arbitrarily. Additionally, in comparative tests, LOBAG-OC has been shown to outperform other popular boundary identification methods [35] such as BIOCLIM [38] and DOMAIN [39].

LOBAG-OC was used to fit an ecological niche model to 20th century point occurrence data on the distribution of An. arabiensis in Africa. This analysis showed that, despite its lower prevalence compared with An. gambiae, An. arabiensis is nevertheless a climate generalist in the sense that it tolerates a wide range of climate conditions. The fit model was then applied to simulated data for three climate change scenarios to forecast the future potential distribution of $A n$. arabiensis. Specifically, the fit niche model was evaluated on maps of climate projections for the year 2050 generated by the Hadley CM 3 model for scenarios A1B, A2A, and B2A. These scenarios derive from the Intergovernmental Panel on Climate Change (IPCC) Special Report on Emissions Scenarios [40]. Scenarios reflect political story lines that emphasize policies aimed at economic considerations (Scenarios $\mathrm{A} 1 \mathrm{~B}$ and $\mathrm{A} 2 \mathrm{~A}$ ) or environmental protection and social equity (Scenario B2A). All scenarios produce ranges for projected global surface warming by 2100 that are intermediate among the scenarios considered by the IPCC [10]. Scenario A1B describes a future world of rapid economic growth, a global population that peaks in mid-century, and rapid introduction of more efficient energy technologies. Scenario A2A envisions a more heterogeneous future world where economic growth and technological change are slower and unevenly distributed. Scenario B2A is a world with more local approaches to environmental sustainability, yielding a slowly increasing global population, intermediate levels of economic development, and less rapid and more diverse technological change than in the A1 scenario [40].

Our model predicts that if any of these three climate change scenarios is realized, the result will be significant reductions in the total land area hospitable

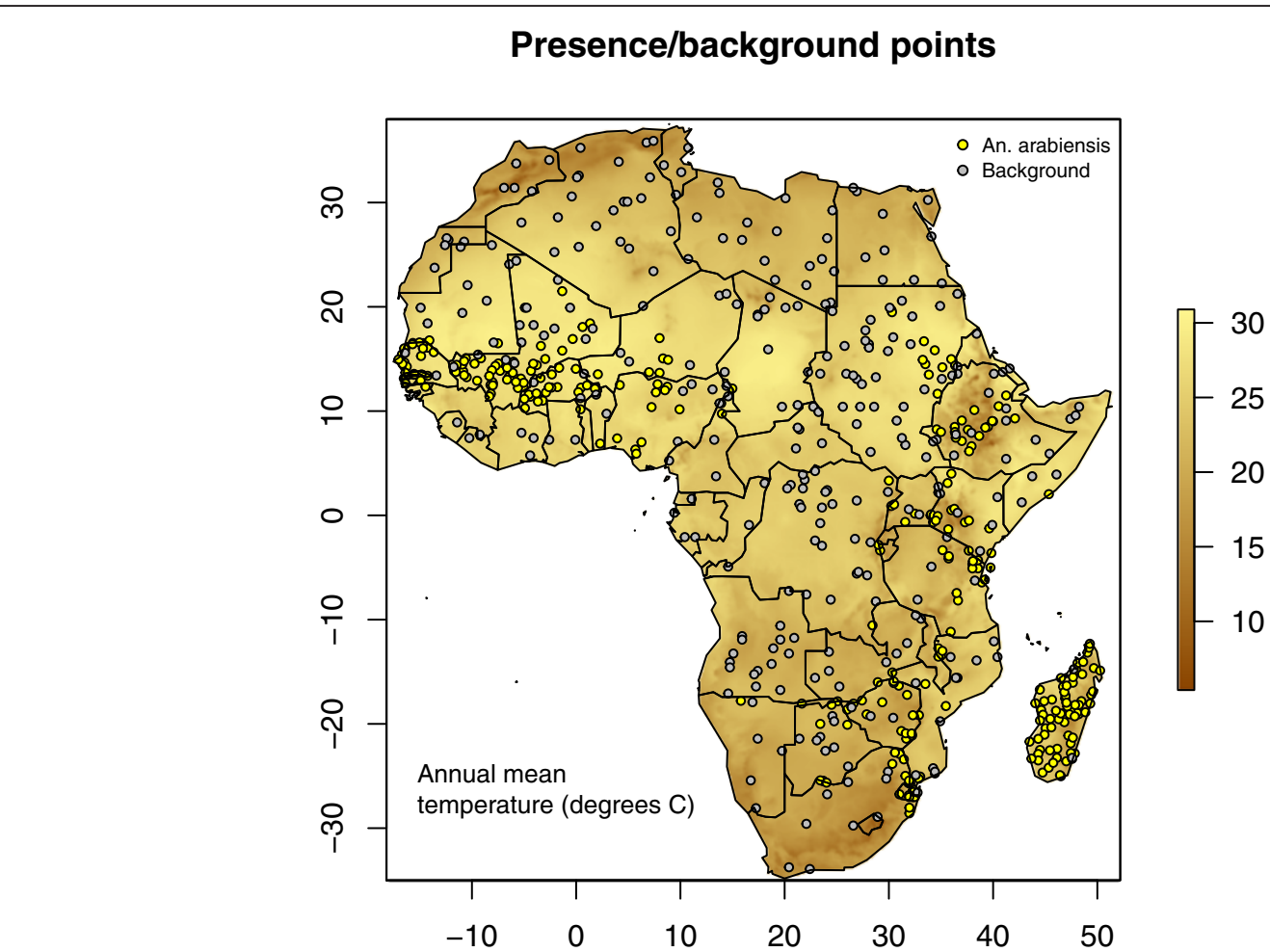

Figure 2 Spatial distribution of Anopheles arabiensis. Distribution of sampling points and a balanced random sample of background points. 
to An. arabiensis. A comparison of modeled current distribution and forecasted potential distribution identified regions where An. arabiensis habitat is lost and regions where it is gained. Map intersections for the three climate change scenarios show that these results are robust to a wide range of assumptions about the future climate system.

\section{Methods}

Data

Occurrence records were obtained by downloading the coordinates of collection sites for An. arabiensis from the Mapping Malaria Risk in Africa data clearinghouse [41-43]. These records reflect collections between 1956 and 1996. Duplicate points were removed and the data thinned so that no more than one record was retained within a distance of 0.5 degrees of another point. The length scale of this thinning depends on latitude, but is around $50 \mathrm{~km}$, which is five to ten times the typical flight distance for a mosquito and a greater distance than is likely to be traveled by an investigator to obtain a convenience sample. Probably, the thinned data are not completely spatially independent, but this thinning should have removed the most egregious multiply sampled populations. This procedure yielded $n_{p}=307$ presence records. Prior to model fitting, the occurrence points were randomly split into training (80\%) and testing (20\%) sets, yielding a total of $\tilde{n}_{p}=246$ observations in the training set. For testing, an additional $\tilde{n}_{p}$ points were randomly selected from the background distribution of environments (Figure 2).

Models were fit to 86 "baseline" environmental features reflecting average conditions for years 1950-2000 (Table 1). Data included 36 interpolated measured variables (average monthly minimum and maximum temperature and precipitation) and the 19 constructed BIOCLIM variables from the WorldClim data set [44]. Raw data for both baseline and forecasted climate were obtained from [45] where they are reported in a 10 minute resolution after statistical downscaling using the delta method [44]. An additional 31 features were constructed, including monthly temperature range, logarithmic transforms of highly skewed variables (monthly precipitation, precipitation in the warmest quarter, and precipitation in the coldest quarter), and empirical cumulative distribution function (ecdf) transforms of some skewed variables (annual precipitation, precipitation in the wettest month, precipitation in the driest month, precipitation in the wettest quarter, and precipitation in the driest quarter). Prior to feature construction, data were clipped to exclude observations not coinciding with continental land regions of Africa or Madagascar. After feature construction, all layers were rescaled by subtracting the mean and dividing by the standard deviation of the baseline data to ensure that
Table 1 Environmental features used to model the potential distribution of An. arabiensis in Africa

\begin{tabular}{|c|c|c|}
\hline Description & Units & $\begin{array}{c}\text { Number of } \\
\text { variables }\end{array}$ \\
\hline \multicolumn{3}{|l|}{ Measured variables } \\
\hline Average monthly minimum temperature & ${ }^{\circ} \mathrm{C}$ & $n=12$ \\
\hline Average monthly maximum temperature & ${ }^{\circ} \mathrm{C}$ & $n=12$ \\
\hline Average monthly precipitation & ${ }^{\circ} \mathrm{C}$ & $n=12$ \\
\hline \multicolumn{3}{|l|}{ BIOCLIM variables } \\
\hline (BIO1) Annual mean temperature & ${ }^{\circ} \mathrm{C}$ & $n=1$ \\
\hline (BIO2) Mean diurnal temperature range & ${ }^{\circ} \mathrm{C}$ & $n=1$ \\
\hline (BIO3) Isothermality & no units & $n=1$ \\
\hline (BIO4) Temperature seasonality & ${ }^{\circ} \mathrm{C}$ & $n=1$ \\
\hline (BIO5) Maximum temperature of warmest month & ${ }^{\circ} \mathrm{C}$ & $n=1$ \\
\hline (BIO6) Minimum temperature of coldest month & ${ }^{\circ} \mathrm{C}$ & $n=1$ \\
\hline (BIO7) Temperature annual range & ${ }^{\circ} \mathrm{C}$ & $n=1$ \\
\hline (BIO8) Mean temperature of wettest quarter & ${ }^{\circ} \mathrm{C}$ & $n=1$ \\
\hline (BIO9) Mean temperature of driest quarter & ${ }^{\circ} \mathrm{C}$ & $n=1$ \\
\hline (BIO10) Mean temperature of warmest quarter & ${ }^{\circ} \mathrm{C}$ & $n=1$ \\
\hline (BIO11) Mean temperature of coldest quarter & ${ }^{\circ} \mathrm{C}$ & $n=1$ \\
\hline (BIO12) Annual precipitation & $\mathrm{mm}$ & $n=1$ \\
\hline (BIO13) Precipitation of wettest month & $\mathrm{mm}$ & $n=1$ \\
\hline (BIO14) Precipitation of driest month & $\mathrm{mm}$ & $n=1$ \\
\hline (BIO15) Precipitation seasonality & $\mathrm{mm}$ & $n=1$ \\
\hline (BIO16) Precipitation of wettest quarter & $\mathrm{mm}$ & $n=1$ \\
\hline (BIO17) Precipitation of driest quarter & $\mathrm{mm}$ & $n=1$ \\
\hline (BIO18) Precipitation of warmest quarter & $\mathrm{mm}$ & $n=1$ \\
\hline (BIO19) Precipitation of coldest quarter & $\mathrm{mm}$ & $n=1$ \\
\hline \multicolumn{3}{|l|}{ Constructed features } \\
\hline Monthly temperature range & ${ }^{\circ} \mathrm{C}$ & $n=12$ \\
\hline log-transforms & $\log \mathrm{mm}$ & $n=14$ \\
\hline ecdf-transforms & no units & $n=5$ \\
\hline
\end{tabular}

both baseline and forecasted data were rescaled to a common range. Features which are ecdf transforms were not rescaled. A principal components analysis was performed to investigate the gross structure of the baseline environmental data.

\section{Model fitting}

Niche modelling was performed using the LOBAG-OC algorithm, a computational approach for modelling ecological niches from presence-only data [35]. LOBAG-OC is an ensemble learning approach for one-class-classification that averages the outcomes of a large number of weakly regularized one-class support vector machines to obtain a numerical value for any given combination of environmental inputs [35]. Briefly, the model fitting algorithm iteratively resamples the original data, fits a one-class 
support vector machine to this sample (a machine learning approach to estimating the support of a statistical distribution, referred to as a base model), and stores the result. A prediction is made by averaging the predictions of the stored base models. By construction, the method is relatively insensitive to irrelevant data or biased sampling, two features that are important for ecological niche modelling. This model has two tuning parameters, $v$, which governs the degree of regularization of the base learners, and the number of votes. As a rule of thumb, LOBAG-OC was shown to perform near optimally with only $2^{6}=64$ votes (but has not been found to diminish in performance as the number of votes increases) and in a large neighborhood of $v$ around $v=2^{-4}$ [35]. The model reported here was fit using 256 votes and $v=2^{-4}$.

LOBAG-OC modelling provides a nonparametric mappable summary of the ecological niche. Generalized boosted regression models $[46,47]$ were applied to a randomly selected subset of 10,000 locations to investigate the relative importance of constituent environmental variables to determining the boundaries of the An. arabiensis geographic range. First, model output was binarized (niche/non-niche) and associations between these labels and 86 environmental covariates were learned using boosted regression trees. The optimal number of trees was selected using fourfold cross-validation. Relative influence was quantified using the method of Breiman [48]. Variables with $>5 \%$ relative influence are reported. An additional 10,000 random locations were inspected to identify differences between baseline and projected conditions (i.e., change from niche to non-niche or change from non-niche to niche) for each climate scenario. Associations between these changes and environmental covariates were identified in the same way.

\section{Results}

Principal components analysis showed the environmental space represented by WorldClim to be relatively low dimensional. Particularly, approximately $55 \%$ of the variation in environmental covariates is contained in just the first two principal components (Figure 3). Plotting of $A n$. arabiensis occurrence points in this space shows that this species is a climate generalist in the sense that it can occur in most of Africa's environmental range (Figure 3). Accordingly, its habitat is fairly widespread throughout Africa, with the main exceptions being the Sahara Desert, the Southern portion of South Africa, and parts of the Congo Basin (Figure 4A). This model was found to have AUC of 0.77 corresponding to sensitivity of 0.93 and specificity of 0.57 at its optimal accuracy of 0.75 . Because these statistics were computed using background points in place of verified absences, these performance statistics represent a lower bound to accuracy. Analysis with boosted regression trees identified maximum December temperature, minimum September temperature, minimum March temperature, precipitation in November, and annual precipitation to be the key variables separating niche from non-niche habitats.

Under three plausible climate change scenarios, the future distribution of $A n$. arabiensis is predicted to be
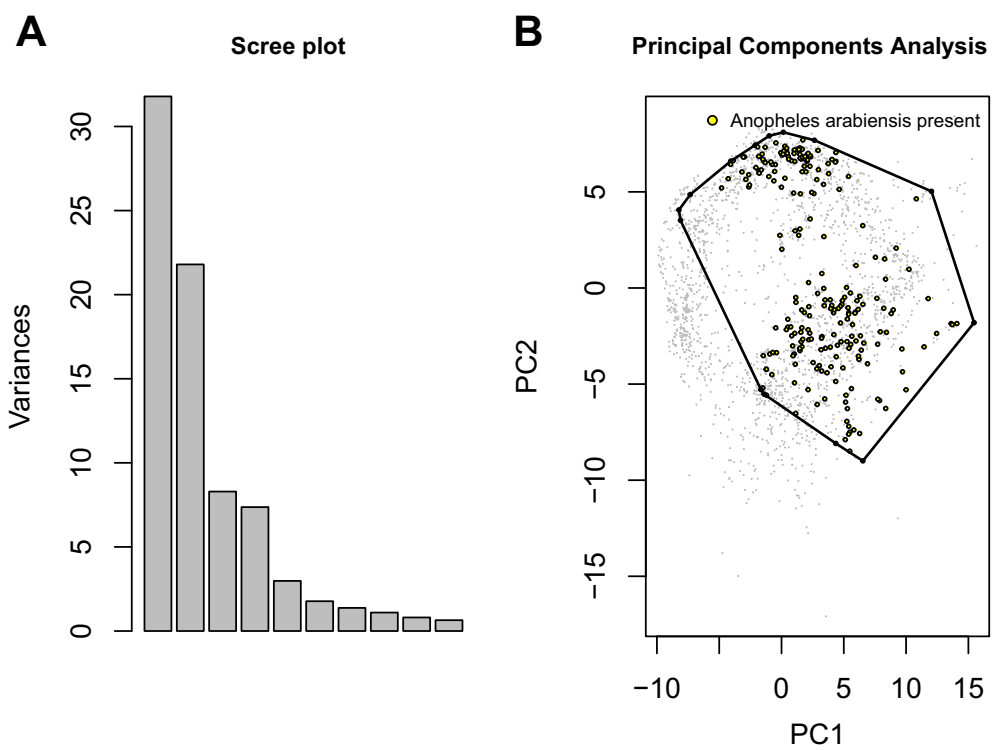

Figure 3 Anopheles arabiensis is found across a broad range of environments. A. Scree plot of the first ten principal components shows that a majority of the environmental variation $(\approx 55 \%)$ may be summarized by the first two principal components. B. Points where Anopheles arabiensis has been collected represented in the space of the first two principal components of the environmental features shows that this species occupies a very large environmental range. 

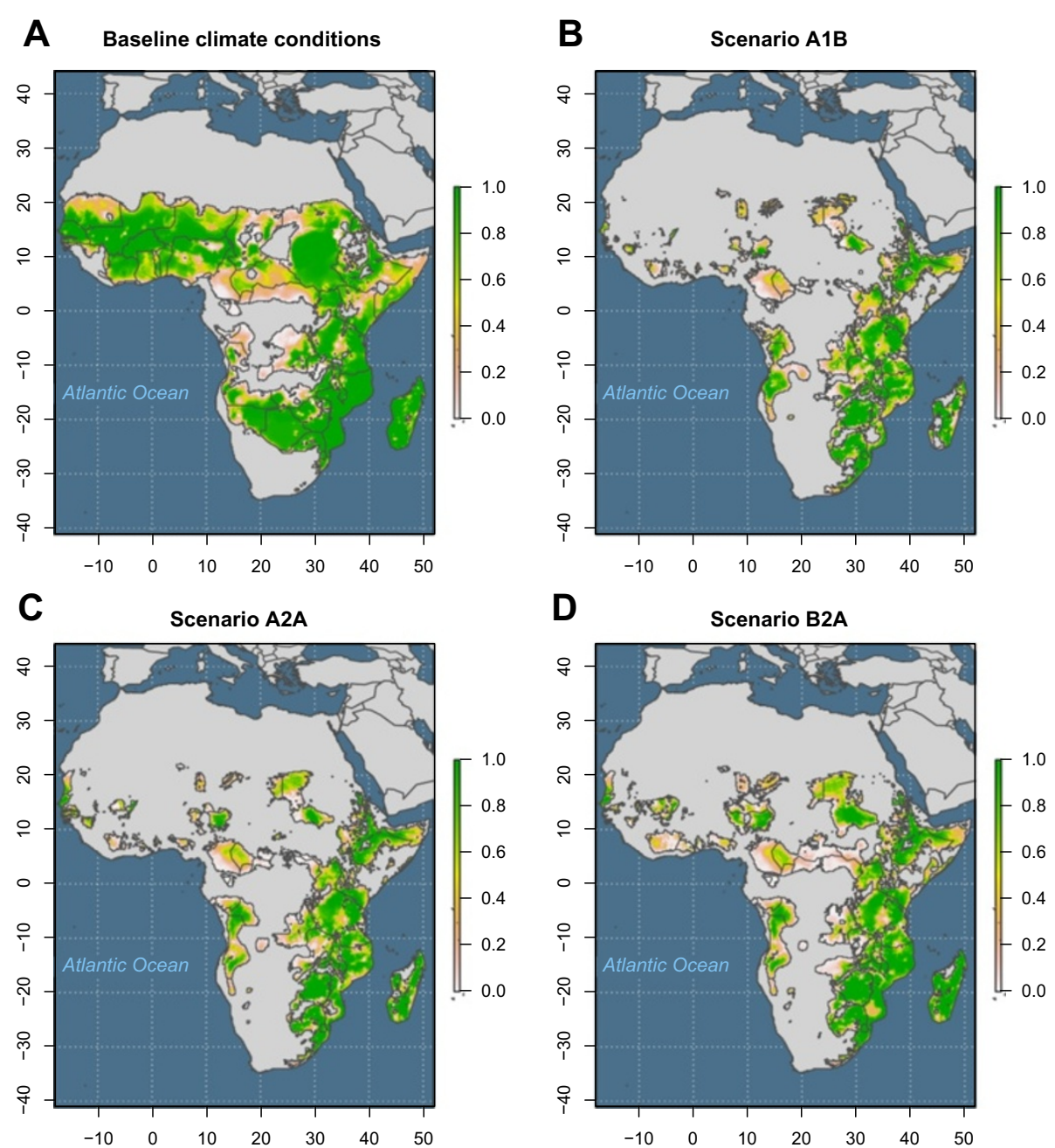

Figure 4 Potential distribution of Anopheles arabiensis under contemporary conditions and three global climate change scenarios. A. Modelled potential distribution of Anopheles arabiensis habitat in Africa given the current global climate. B. Future potential distribution of Anopheles arabiensis in Africa under IPCC Scenario A1B. C. Future potential distribution of Anopheles arabiensis in Africa under IPCC Scenario A2A. D. Future potential distribution of Anopheles arabiensis in Africa under IPCC Scenario B2A.

considerably reduced (Figure 4). Figure 5 shows the total geographic area (sq. km) inhabitable by An. arabiensis under both baseline and forecasted future climate conditions and relative to the area of the entire Africa land mass. These results suggest that even in the absence of vector control and land conversion, the spatial distribution and total exposure of the African population to malaria transmitted by $A n$. arabiensis is expected to change dramatically. Indeed, while the estimated effect of projected climate change is relatively large (reductions in area of 48\%-61\%), the differences among climate change scenarios are relatively small (Figure 4B-4D). Map differences between baseline and projected climate models suggest that reductions of habitat will be especially extensive in Western and Central Africa; portions of Botswana, Namibia, and Angola in Southern Africa; and portions of Sudan, South Sudan, Somalia, and Kenya in East Africa (Figure 6). The East African Rift Valley and Eastern Coast of Africa, where An. arabiensis is most abundant today, are expected to remain habitable. There will be some modest gains in habitat, especially on the margins of the current range in South Sudan, South Africa, and Angola. The key variables driving change in habitat were associated with temperature and precipitation from November to March. Particularly, maximum December temperature, maximum January temperature, precipitation in November, minimum March temperature, and minimum November temperature explain most of the difference between baseline and scenario A1B projections. All of these except minimum November temperature were important to the difference between baseline and scenario A2A and scenario B2A projections. Thus, it appears that 


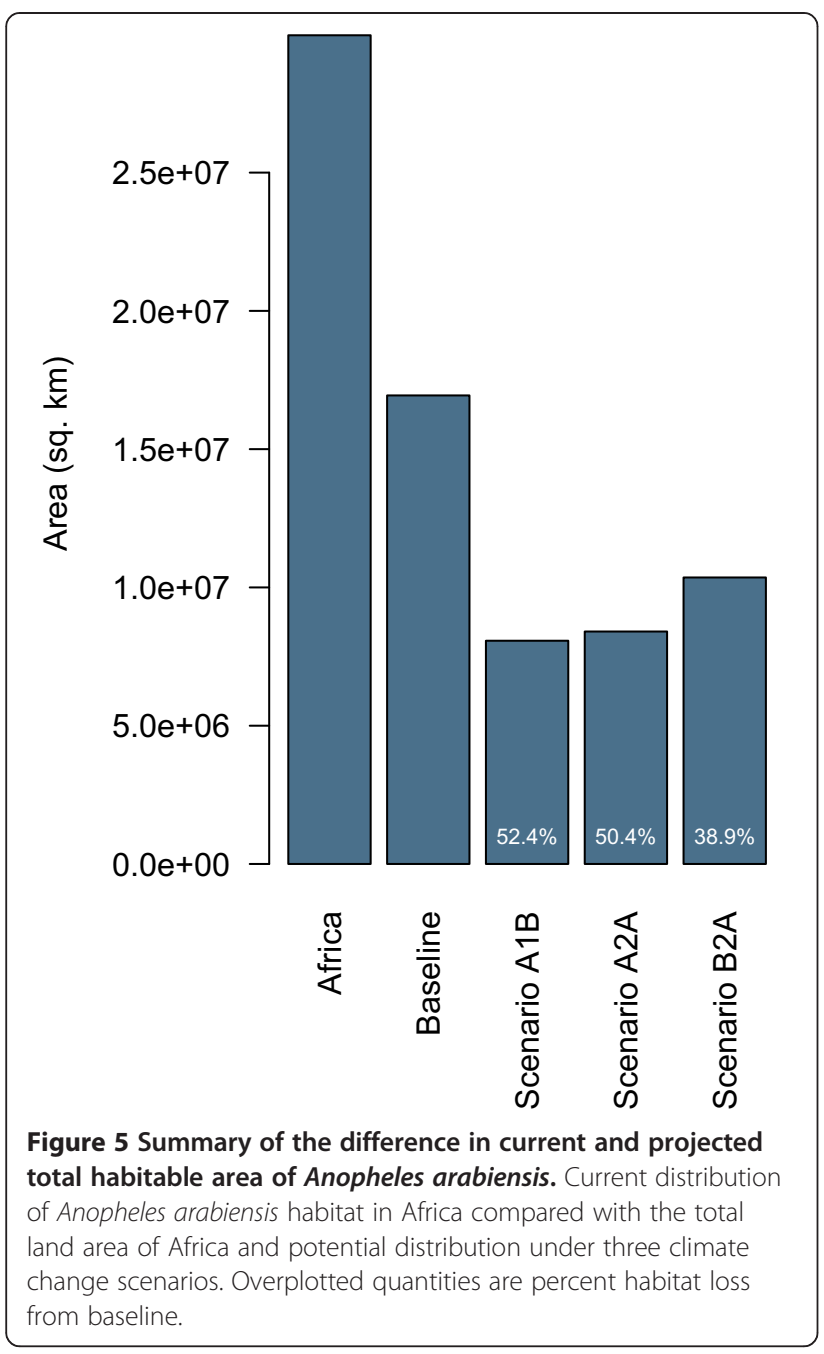

the primary drivers of environmental change concern dry season climate, possibly interacting with the East African short rains.

Given the inherent uncertainty about the effectiveness of present and future climate policies and the contingent scientific uncertainties that obtain at the present time, exactly what trajectory the future climate will take remains a major continuing unknown. An important question, therefore, is how sensitive are these projected gains and losses of An. arabiensis habitat to the details of the climate scenarios for which they are computed. To address this issue of the robustness of our projections, agreement among the three scenarios was calculated. First, for each pixel the number of scenarios for which it was predicted to be habitable by An. arabiensis was calculated (Figure 7A). Then, for those locations in which all scenarios were in agreement, gains (Figure 7B) were disaggregated from losses (Figure 7C). These results show that for those locations where a change of habitability is predicted, all three scenarios agree in the large majority of cases. Of these, the overwhelming majority predict loss of habitat.

\section{Discussion}

This study showed An. arabiensis to be a climate generalist with widespread potential distribution in Africa. The map of the current potential distribution of $A n$. arabiensis is in broad agreement with other published maps $[29,30,49]$ disagreeing, as expected, primarily at the margins and extreme interior (i.e., Congo and Ogooué basins). An ecological niche model for An. arabiensis projected on data from global climate simulations predicts that despite these wide tolerances, the potential distribution of $A n$. arabiensis is likely to be reduced by $48 \%-61 \%$ by 2050 (Figure 5 ).

These results are important for interpreting the effectiveness of ongoing campaigns to eliminate malaria in several parts of Africa. First, not all reductions in malaria burden should be attributed to elimination campaigns. The signature of climate on An. arabiensis distribution that was detected here may at least partly explain other declines in prevalence in sub-Saharan Africa, for instance on Pemba and in mainland Tanzania [8,9]. Second, as indoor malaria-control activities, such as IRS and the distribution of ITNs, increase in effectiveness, one should expect a greater proportion of infections to be acquired out of doors. As a result there will be diminishing returns to increasing malaria elimination efforts. This does not mean that such increases would be unwarranted or ineffective. To the contrary, reducing such efforts short of complete elimination is an invitation for resurgence. The current results may be useful just insofar as they highlight regions in which malaria is most likely to be acquired from and maintained by $A n$. arabiensis.

A long view of malaria elimination should therefore strategically consider the future climate of Africa and deploy interventions accordingly. Several strategies might be recommended. Because malaria is predicted to persist longest in those regions that remain habitable to An. arabiensis after climate change, it may be expected that these will be the most difficult regions from which to eliminate malaria, and that these will be the sources of any resurgence. From one point of view, these then are the regions that should be targeted most intensively with methods for reducing indoor biting, i.e., ITNs and IRS. The rationale for this strategy is that to eliminate malaria requires reducing transmission everywhere (to remove sources for resurgence). However, to reduce transmission in regions where outdoor biting is considerable, i.e., regions where $A n$. arabiensis will persist, requires proportionately greater reductions in indoor biting to compensate. An alternative strategy considers the future potential distribution of An. arabiensis to be relatively minimal already. If elimination can be achieved outside 

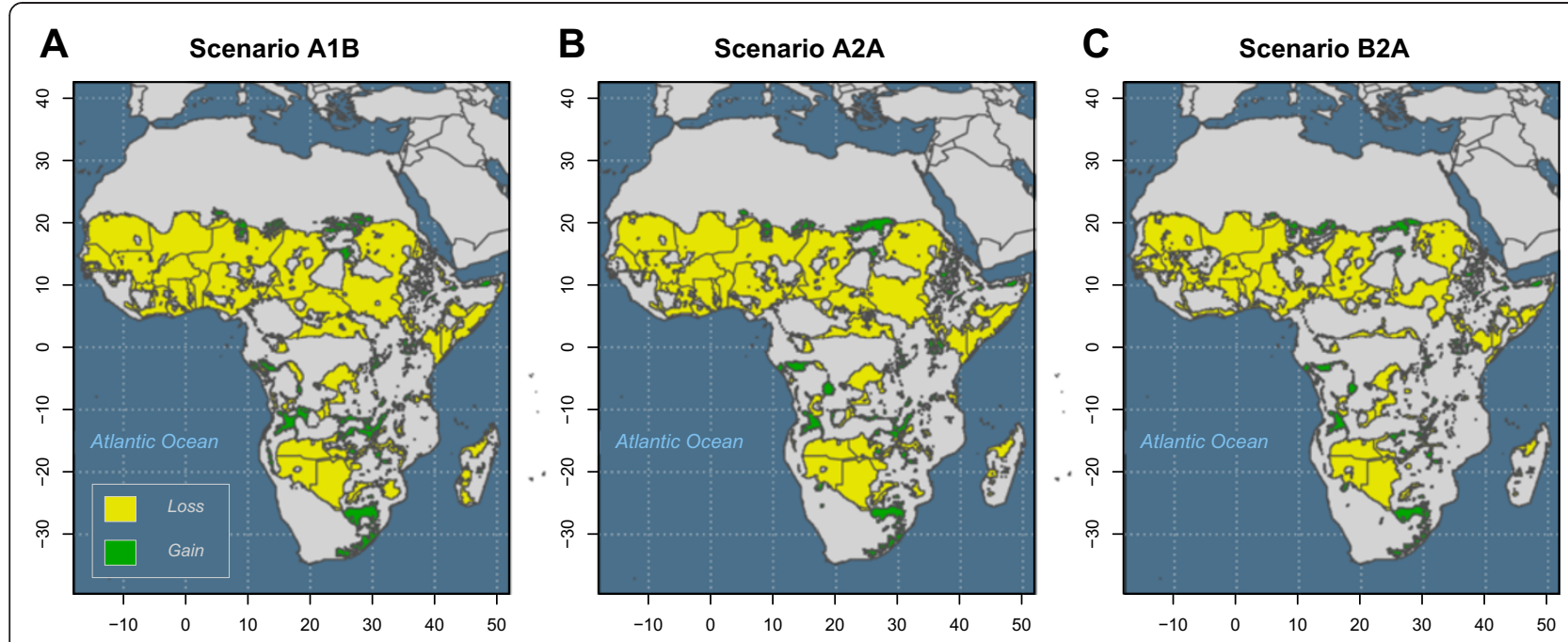

Figure 6 Differences between the current and projected distribution of Anopheles arabiensis. A. Losses and gains of Anopheles arabiensis habitat in Africa under future climate scenario A1B compared with the current distribution. B. Losses and gains of Anopheles arabiensis habitat in Africa under future climate scenario A2A compared with the current distribution. C. Losses and gains of Anopheles arabiensis habitat in Africa under future climate scenario B2A compared with the current distribution.

this region, then subsequent effort might be best concentrated in a cordon sanitaire to limit the potential for reintroduction and resurgence. Finally, a hybrid strategy might exploit the fact that the predicted future potential distribution of An. arabiensis is both minimal and fragmented. Since fragmented populations are most vulnerable to extinction, it would possibly be most effective to try a mixed strategy: isolate core infected areas to prevent reinfection and break up regions of transmission at their most vulnerable points. In any case, it would seem advisable to consider the probable future distribution of An. arabiensis in the design of malaria elimination strategies. How to optimally combine these strategies remains, to our knowledge, an open problem.

Our work raises a number of questions about disease risk mapping. The introduction to this paper argues on conceptual grounds that the potential distribution of a vector species is better identified using boundary estimation techniques than methods for classification (i.e., methods that aim to discriminate presence from absence
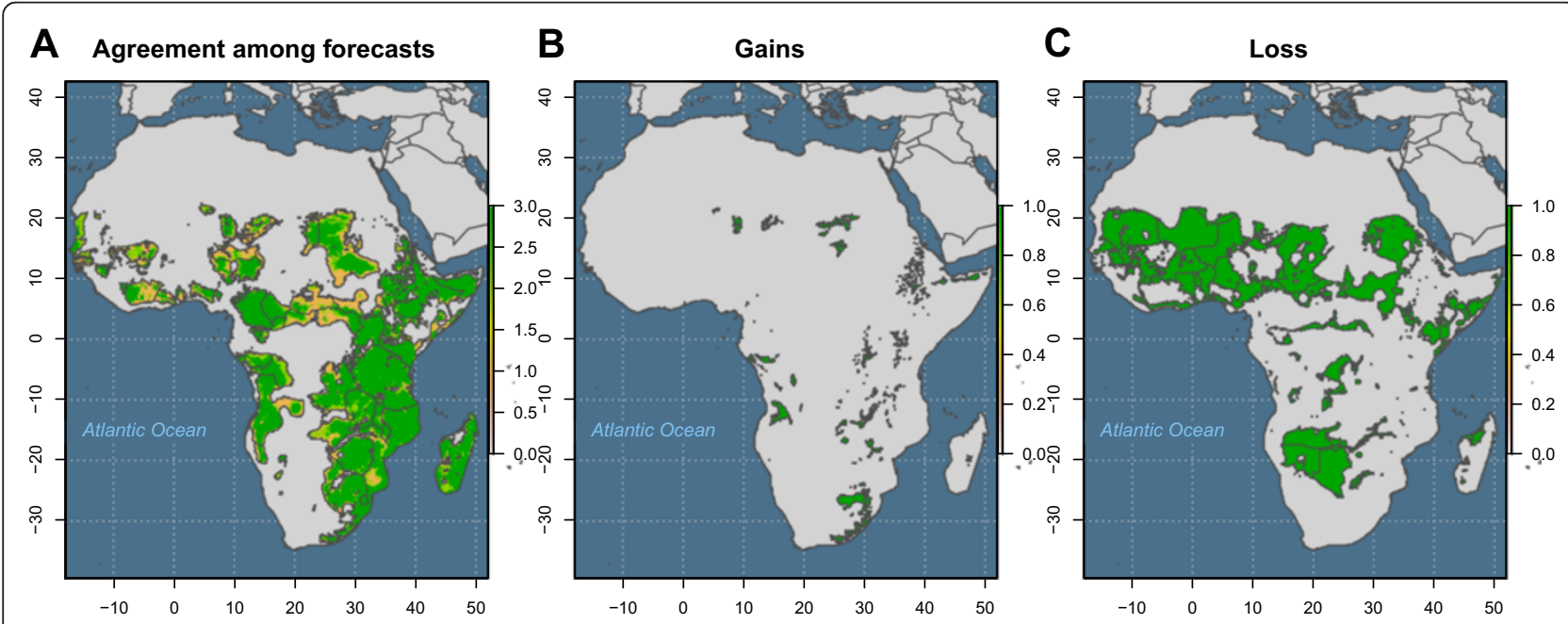

Figure 7 Projected distribution of Anopheles arabiensis is robust to variations in climate change scenario. A. Number of scenarios in which Anopheles arabiensis habitat is predicted to be lost or gained (grey: no scenario predicts habitat; orange: habitat predicted under one climate change scenario; light green: habitat predicted under two climate change scenarios; green: habitat predicted under all three climate change scenarios). B. Universal agreement among three climate change scenarios that An. arabiensis habitat will be gained. C. Universal agreement among three climate change scenarios that An. arabiensis habitat will be lost. 
points or occurrence points from the background distribution of environments). A simple numerical example in two dimensions shows why this is the case. The current study is the first major application of a new method, LOBAG-OC, for boundary estimation of ecological niche models. Although earlier results showed LOBAG-OC to perform comparably to other methods, such as MAXENT, and to be robust to several tuning parameters, how to optimize LOBAG-OC remains an important problem for further study.

Finally, not all determinants of a species habitat are captured by the climate variables encoded in the WorldClim data set. Although these results are expected to be robust with respect to the environmental variables that determine the range limits of An. arabiensis, these data and this model were not designed for and are not expected to explain local variation in abundance. The spatial resolution at which the current study was performed is therefore not suited to quantifying local variation in human exposure or health risk. Thus, additional regionally targeted modelling exercises will be of importance for tactically guiding interventions. Such models should make particular use of local information on human population density and land cover [28]. Our study, in contrast, was designed to produce a coarse-grained picture of the $A n$. arabiensis distribution at the continental scale. Given the correspondingly grand scale of current investments in elimination, this scale may indeed be the most suitable to designing effective malaria policies.

\section{Conclusion}

The future distribution of African malaria is predicted to be more dependent on the distribution of An. arabiensis and environmental variables than the current distribution. Further, presence-background methods for modelling species distributions may be expected to yield results with unnecessarily high Type I error, higher than that of suitably chosen boundary identification methods. One presenceonly method, LOBAG-OC, predicts that the total area habitable to An. arabiensis will be reduced by $48 \%-61 \%$ by the year 2050 due to changes in the global climate system. Both the magnitude and spatial distribution of this reduction appear to be robust to the choice of climate scenario. How to maximally exploit this ecological relationship in malaria control and elimination is now an important question for research.

\section{Competing interests}

The authors declare that they have no competing interests.

\section{Authors' contributions}

JMD developed the model and performed the analysis. JMD and JCB jointly wrote the manuscript. Both authors read and approved the final manuscript.

\section{Acknowledgements}

The authors thank A. Kramer for comments on an earlier draft of this paper. This study was supported by a grant from the National Institutes of Health (R01GM093345).

\section{Author details}

${ }^{1}$ Odum School of Ecology, University of Georgia, 140 E Green Street, 30602-2202 Athens, GA, USA. ²Department of Public Health Sciences, University of Miami Miller School of Medicine, 1120 NW 14th Street, 33136 Miami, FL, USA.

Received: 4 December 2013 Accepted: 27 May 2014

Published: 3 June 2014

\section{References}

1. Gething PW, Smith DL, Patil AP, Tatem AJ, Snow RW, Hay SI: Climate change and the global malaria recession. Nature 2010, 465:342-345.

2. O'Meara WP, Mangeni JN, Steketee R, Greenwood B: Changes in the burden of malaria in sub-Saharan Africa. Lancet Infect Dis 2010, 10:545-555.

3. Snow RW, Guerra CA, Noor AM, Myint HY, Hay SI: The global distribution of clinical episodes of Plasmodium falciparum malaria. Nature 2005, 434:214-217.

4. Cibulskis RE, Aregawi M, Williams R, Otten M, Dye C: Worldwide incidence of malaria in 2009: estimates, time trends, and a critique of methods. PLOS Med 2011, 8:1001142.

5. Hay SI, Okiro EA, Gething PW, Patil AP, Tatem AJ, Guerra CA, Snow RW: Estimating the global clinical burden of Plasmodium falciparum malaria in 2007. PLoS Med 2010, 7:1000290.

6. World Health Organisation: World Malaria Report 2013. Geneva: 2013.

7. White NJ: Intermittent presumptive treatment for malaria. PLoS Med 2005, 2:e3.

8. Jaenisch T, Sullivan DJ, Dutta A, Deb S, Ramsan M, Othman MK, Gaczkowski $\mathrm{R}$, Tielsch J, Sazawal S: Malaria incidence and prevalence on Pemba island before the onset of the successful control intervention on the Zanzibar archipelago. Malar J 2010, 9:32.

9. Meyrowitsch DW, Pedersen EM, Alifrangis M, Scheike TH, Malecela MN, Magesa SM, Derua YA, Rwegoshora RT, Michael E, Simonsen PE: Is the current decline in malaria burden in sub-Saharan Africa due to a decrease in vector population? Malar J 2011, 10:188.

10. Intergovernmental Panel on Climate Change: Summary for policymakers. In Climate Change 2007: The Physical Science Basis. Contribution of Working Group I to the Fourth Assessment Report of the Intergovernmental Panel on Climate Change. Cambridge: Cambridge University Press; 2007:1-18.

11. Martens WJ, Niessen LW, Rotmans J, Jetten TH, McMichael AJ: Potential impact of global climate change on malaria risk. Environ Health Perspect 1995, 103:458-464.

12. Patz JA: Global climate change and emerging infectious diseases. J Am Med Assoc 1996, 275:217.

13. Reiter P: Climate change and mosquito-borne disease. Environ Health Perspect 2001, 109:141-161.

14. Hay SI, Cox J, Rogers DJ, Randolph SE, Stern DI, Shanks GD, Myers MF, Snow RW: Climate change and the resurgence of malaria in the East African highlands. Nature 2002, 415:905-909.

15. McMichael AJ, Woodruff RE, Hales S: Climate change and human health: present and future risks. Lancet 2006, 367:859-869.

16. Patz JA, Campbell-Lendrum D, Holloway T, Foley JA: Impact of regional climate change on human health. Nature 2005, 438:310-317.

17. Dobson A: Climate variability, global change, immunity, and the dynamics of infectious diseases. Ecology 2009, 90:920-927.

18. Lafferty KD: The ecology of climate change and infectious diseases. Ecology 2009, 90:888-900.

19. Ostfeld RS: Climate change and the distribution and intensity of infectious diseases. Ecology 2009, 90:903-905.

20. Pascual M, Bouma MJ: Do rising temperatures matter? Ecology 2009, 90:906-912.

21. Fuller DO, Parenti MS, Hassan AN, Beier JC: Linking land cover and species distribution models to project potential ranges of malaria vectors: an example using Anopheles arabiensis in Sudan and Upper Egypt. Malar J 2012, 11:264. 
22. Derua YA, Alifrangis M, Hosea KM, Meyrowitsch DW, Magesa SM, Pedersen EM, Simonsen PE: Change in composition of the Anopheles gambiae complex and its possible implications for the transmission of malaria and lymphatic filariasis in north-eastern Tanzania. Malar J 2012, 11:188.

23. Mwangangi JM, Mbogo CM, Orindi BO, Muturi EJ, Midega JT, Nzovu J, Gatakaa H, Githure J, Borgemeister C, Keating J, Beier JC: Shifts in malaria vector species composition and transmission dynamics along the Kenyan coast over the past 20 years. Malar J 2013, 12:13.

24. Sinka ME, Bangs MJ, Manguin S, Coetzee M, Mbogo CM, Hemingway J, Patil AP, Temperley WH, Gething PW, Kabaria CW, Okara RM, van Boeckel T, Godfray HCJ, Harbach RE, Hay SI: The dominant Anopheles vectors of human malaria in Africa, Europe and the Middle East: occurrence data, distribution maps and bionomic precis. Parasit Vectors 2010, 3:117.

25. Jones CM, Toé A, Dabiré HK, Sanou A, Namountougou M, Hughes A Diabaté $\mathrm{R}$, Simard $\mathrm{F}$, Ranson $\mathrm{H}$ : Additional selection for insecticide resistance in urban malaria vectors: DDT resistance in Anopheles arabiensis from Bobo-Dioulasso, Burkina Faso. PLoS One 2012, 7:45995.

26. Kitau J, Oxborough RM, Tungu PK, Matowo J, Malima RC, Magesa SM, Bruce J, Mosha FW, Rowland MW: Species shifts in the Anopheles gambiae complex: do LLINs successfully control Anopheles arabiensis? PLoS One 2012, 7:31481.

27. Stuckey EM, Stevenson JC, Cooke MK, Owaga C, Marube E, Oando G, Hardy D, Drakeley C, Smith TA, Cox J, Chitnis N: Simulation of malaria epidemiology and control in the highlands of Western Kenya. Malar $J$ 2012, 11:357.

28. Ayala D, Costantini C, Ose K, Kamdem GC, Antonio-Nkondjio C, Agbor J-P, Awono-Ambene P, Fontenille D, Simard F: Habitat suitability and ecological niche profile of major malaria vectors in Cameroon. Malar J 2009, 8:307.

29. Lindsay SW, Parson L, Thomas CJ: Mapping the ranges and relative abundance of the two principal African malaria vectors, Anopheles gambiae sensu stricto and $A n$. arabiensis, using climate data. Proc $R$ Soc $B$ 1998, 265:847-854

30. Moffett A, Shackelford N, Sarkar S: Malaria in Africa: vector species' niche models and relative risk maps. PLoS One 2007, 2:824.

31. Elith J, Leathwick JR: Species distribution models: ecological explanation and prediction across space and time. Annu Rev Ecol Evol Syst 2009, 40:677-697.

32. Phillips SJ, Dudík M, Elith J, Graham CH, Lehmann A, Leathwick J, Ferrier S: Sample selection bias and presence-only distribution models: implications for background and pseudo-absence data. Ecol Appl 2009, 19:181-197.

33. Phillips SJ, Dudík M, Schapire RE: A maximum entropy approach to species distribution modeling. In Twenty-first International Conference on Machine Learning ICML 04. New York, NY USA: Association for Computing Machinery; 2004:655-662.

34. Elith J, Phillips SJ, Hastie T, Dudík M, Chee YE, Yates CJ: A statistical explanation of MaxEnt for ecologists. Diversity Distrib 2011, 17:43-57.

35. Drake JM: Ensemble algorithms for ecological niche modeling from presence-background and presence-only data. Ecosphere. in press.

36. Hirzel AH, Hausser J, Chessel D, Perrin N: Ecological-niche factor analysis: how to compute habitat-suitability maps without absence data? Ecology 2002, 83:2027-2036.

37. Renner IW, Warton DI: Equivalence of MAXENT and Poisson point process models for species distribution modeling in ecology. Biometrics 2013, 69:274-281.

38. Booth TH, Nix HA, Busby JR, Hutchinson MF: Bioclim: the first species distribution modelling package, its early applications and relevance to most current MaxEnt studies. Diversity Distrib 2014, 20:1-9.

39. Carpenter G, Gillison AN, Winter J: DOMAIN: a flexible modelling procedure for mapping potential distributions of plants and animals. Biodivers Conserv 1993, 2:667-680

40. Nakicenovic N, Alcamo J, Davis G, de Vries B, Fenhann J, Gaffin S, Gregory K, Grubler A, Jung TY, Kram T, La Rovere EL, Michaelis L, Mori S, Morita T, Pepper W, Pitcher HM, Price L, Riahi K, Roehrl A, Rogner H-H, Sankovski A, Schlesinger M, Shukla P, Smith SJ, Swart R, van Rooijen S, Victor N, Dadi Z: Special Report on Emissions Scenarios: a special report of Working Group III of the Intergovernmental Panel on Climate Change. Cambridge: Cambridge University Press; 2000.

41. Le Sueur D, Binka F, Lengeler C, de Savigny D, Snow B, Teuscher T, Toure Y: An atlas of malaria in Africa. Africa Health 1997, 19:23-24.
42. Craig MH, Snow RW, le Sueur D: A climate-based distribution model of malaria transmission in sub-Saharan Africa. Parasitol Today 1999, 15:105-111.

43. Hay SI, Snow RW: The Malaria Atlas Project: developing global maps of malaria risk. PLoS Med 2006, 3:473.

44. Hijmans RJ, Cameron SE, Parra JL, Jones PG, Jarvis A: Very high resolution interpolated climate surfaces for global land areas. Int J Climatol 2005, 25:1965-1978.

45. Downscaled GCM data portal. [http://www.ccafs-climate.org/data/]

46. Friedman $\mathrm{JH}$ : Greedy function approximation: a gradient boosting machine. Ann Stat 2001, 29:1189-1232.

47. Ridgeway G: gbm: generalized boosted regression models. [http://cran. r-project.org/package $=$ gbm]

48. Breiman L: Random forests. Mach Learn 2001, 45:5-32.

49. Sinka ME, Bangs MJ, Manguin S, Rubio-Palis Y, Chareonviriyaphap T, Coetzee M, Mbogo CM, Hemingway J, Patil AP, Temperley WH, Gething PW, Kabaria CW, Burkot TR, Harbach RE, Hay SI: A global map of dominant malaria vectors. Parasit Vectors 2012, 5:69.

doi:10.1186/1475-2875-13-213

Cite this article as: Drake and Beier: Ecological niche and potential distribution of Anopheles arabiensis in Africa in 2050. Malaria Journal 2014 13:213.

\section{Submit your next manuscript to BioMed Central and take full advantage of:}

- Convenient online submission

- Thorough peer review

- No space constraints or color figure charges

- Immediate publication on acceptance

- Inclusion in PubMed, CAS, Scopus and Google Scholar

- Research which is freely available for redistribution 